\title{
Duplicación de prótesis total. Descripción de un caso*
}

\author{
Denture Duplication. Case Report
}

Duplicação de dentadura. Relato de caso

Fecha de recepción: 27-02-2018 | Fecha de aceptación: 03-12/2019

\begin{abstract}
JimMY MATiz Cuervo ${ }^{a}$
Universidad Nacional de Colombia, Bogotá, Colombia. jmatizc@unal.edu.co. https://orcid.org/0000-0001-5180-6555
\end{abstract}

*Descripción de caso

${ }^{a}$ Correspondencia: jmatizc@unal.edu.co

doi: $\underline{\text { https://doi.org/10.11144/Javeriana.uo38-81.dptd }}$

Cómo citar: Matiz Cuervo J. Duplicación de prótesis total. Descripción de un caso. Univ Odontol. 2019 jul-dic; 38(81). https://doi.org/10.11144/Javeriana.uo38-81.dptd

\section{RESUMEN}


Antecedentes: Tradicionalmente, para elaborar una prótesis total se ha llevado a cabo una serie de pasos que continúan siendo esenciales a pesar del avance tecnológico. Sin embargo, en algunos pacientes es difícil adaptar prótesis nuevas. Objetivo: Describir una técnica de duplicación de prótesis totales elaboradas en acrílico de termocurado en la que se simplifican los pasos. Esta técnica busca preservar los mecanorreceptores intraorales y la adaptación muscular que un paciente ha manejado por años con sus prótesis antiguas. Descripción del caso: Un paciente con reabsorción ósea avanzada en ambos maxilares necesitaba cambiar sus prótesis de más de 20 años. Se elaboraron duplicadores similares a los de $3 \mathrm{i}{ }^{\circledR}$ en los que se obtuvieron copias similares a las del paciente. Se montaron y terminaron con impresión a boca cerrada simultáneamente de ambos maxilares. Se obtuvieron prótesis definitivas en termocurado con bases similares a las del paciente manteniendo la fisiología neurosensorial, de tal forma que no sintiera el cambio. Conclusiones: La conservación del sistema de sensorreceptores de tacto, frio, presión, calor y actividad muscular es fundamental para el éxito en casos de duplicación protésica. El autor tiene alrededor de 2500 casos de duplicación de dentaduras en una práctica de más de 20 años.

\section{Palabras clave}

dentaduras; duplicación protésica; manejo protésico, mecanorreceptores intraorales; odontología; prostodoncia; prótesis total; rehabilitación oral

\section{ABSTRACT}

Background: Traditionally, a series of steps have been carried out to make a complete denture, which are still essential despite technological advances. However, in some patients it is difficult 
to adapt new dentures. Objective: To describe a simplified technique for the duplication of total dentures using thermo-cured acrylic. This technique seeks to preserve intraoral mechanoreceptors and muscle adaptation that a patient has managed for years with the old dentures. Case description: A patient with severe bone resorption in both jaws needed to change 20-plus-yearold dentures. Duplicators similar to $3 \mathrm{i}{ }^{\circledR}$ were made to obtain copies similar to the patient's dentures. Simultaneous impressions of both jaws with closed mouth were taken. The definitive thermo-cured dentures similar to the patient's old ones maintained neurosensory physiology and the patient did not feel the change. Conclusions: Conservation of the sensory receptors such as cold, pressure, heat and muscle activity is essential for success in cases of denture duplication. The author has around 2500 cases of denture duplication in a more than-20-year practice.

\section{Keywords}

dentistry; dentures; denture duplication; intraoral mechanoreceptors; oral rehabilitation; prosthetic management; prosthodontics; total dentures

\section{RESUMO}

Antecedentes: Tradicionalmente, para desenvolver uma prótese total, foram executadas uma série de etapas que continuam sendo essenciais, apesar do progresso tecnológico. No entanto, em alguns pacientes, é difícil adaptar novas próteses. Objetivo: Descrever uma técnica para duplicar próteses totais de acrílico termocurável, na qual as etapas são simplificadas. Essa técnica busca preservar os mecanorreceptores intraorais e a adaptação muscular que um paciente administra há anos com suas próteses antigas. Descrição do caso: Um paciente com reabsorção óssea avançada nas duas mandíbulas precisava trocar suas próteses por mais de 20 anos. Duplicadores semelhantes aos de 3i® foram feitos 
nos quais foram obtidas cópias semelhantes às do paciente. Eles foram montados e finalizados com impressão de boca fechada simultaneamente dos dois maxilares. As próteses termocurativas definitivas foram obtidas com bases semelhantes às do paciente que mantinha fisiologia neurossensorial, para que ele não sentisse a mudança. Conclusões: A preservação do sistema sensor, sensor de frio, pressão, calor e atividade muscular é essencial para o sucesso em casos de duplicação protética. O autor tem cerca de 2.500 casos de duplicação de próteses em uma prática de mais de 20 anos.

\section{Palavras chave}

dentaduras; duplicação protética; manejo protético; mecanorreceptores intraorais; odontologia; prótese dentária; prótese total; reabilitação oral

\section{INTRODUCCIÓN}

El número de personas mayores de 90 y 100 años de edad en varias partes del mundo ha ido en aumento, lo cual tiene múltiples implicaciones en la salud oral como reabsorción ósea severa, fragilidad de las mucosas, poca coordinación muscular, perdida de estabilidad oclusal y dificultad de adaptación a unas prótesis nuevas es cada vez más difícil (1). Los cambios fisiológicos normales asociados a la edad (mucosa menos fibrosa, fragilidad capilar, fragilidad de mucosas, menos tejido queratinizado, y menos resistencia a las cargas físicas y químicas, entre otras) resultan en condiciones orales desfavorables desde el punto de vista rehabilitador en los pacientes de más edad (2).

El éxito protésico se fundamenta en el manejo adecuado de varios aspectos: adsorción, absorción, humedad, tensión superficial, fuerza capilar, espesor de la saliva, viscosidad, presión atmosférica, 
gravedad, adaptación de la base, sellado periférico, cohesión, vacío, control muscular, peso y calidad del tejido (3). Otro aspecto primordial es la calidad de los tejidos que recubren los maxilares que tienen con un biotipo fuerte y un tejido de recubrimiento grueso (epitelio queratinizado), que determinan una adaptación más fácil a prótesis nuevas que un tejido friable y delgado. Además, cuando se elabora una prótesis nueva, generalmente hay cambios notables con respecto a las prótesis que ha estado utilizando el paciente porque, a pesar de tener las dimensiones educadas y de haberse realizado con la mejor técnica posible, algunos pacientes resienten el cambio y no se adaptan a las nuevas prótesis (4)

Sobre esta técnica protésica existen referencias que datan de las décadas de 1950 (de investigadores como Adam y Geiger), 1960 (autores como Chick, Scher, Chamberlain, Anderson, Shaw y Manoli), 1970 (por ejemplo, Cooper, Storer, Zoeller, Staffort, Huggett, Boss, Duthie, Azarmehr, Singer y Wagner), 1980 (estudios de Heath, Davenport, Dukes, Nassif, Brewer) y unos pocos de la década se 1990 (McCord, Kippax, Ansari, Assery, Lindquist, Morrow). La experiencia del autor de este artículo comienza en 1998 (1-23).

Se debe aprovechar la adaptación protésica que tiene el paciente a unas prótesis antiguas. Sobre ellas se trabaja, en primer lugar, para obtener unas nuevas prótesis con una base protésica casi exacta a las anteriores. En segundo lugar, se busca actuar sobre los mecanorreceptores intraorales para no cambiar sus umbrales de estímulo después de 10,15 o 25 años de experiencia protésica (5). Con la técnica de duplicación protésica se pretende obtener cambios mínimos de presión muscular, longitud y tensión muscular. No se quiere tampoco modificar, en lo posible, los receptores de la actividad motora, ni los mecanorreceptores (terminaciones nerviosas libres) (5). Igualmente, se quiere que los parámetros fisiológicos de la masticación, como ciclos, frecuencia, intensidad y duración, se mantengan lo más 
cercano a la cotidianidad del paciente de tal forma que la información sensorial de la cavidad oral se mantenga estable y la respuesta de las motoneuronas sea similar a la fisiología cotidiana del paciente. Al usarse esta técnica no se deben olvidar fundamentos como la determinación de la dimensión vertical, la elección de una técnica especifica de enfilado dental y los determinantes de la oclusión, que son los que determinan en parte el tipo de dientes artificiales a utilizar (6). La duplicación tiene ventajas que tienen que ver con la rápida obtención de la base protésica y del enfilado. Asimismo, y las ventajas biológicas se relacionan con el aprovechamiento de la adaptación muscular y de los propioceptores a las prótesis antiguas (7).

Esta técnica puede ser utilizada en pacientes que no han tenido éxito con las técnicas convencionales, con mala experiencia protésica, que han usado prótesis por 20, 30 o incluso 40 años, con discrepancias marcadas entre los arcos maxilares, con reabsorción ósea severa, y postrados o en cama por enfermedades sistémicas limitantes (8-10). Su uso desde hace más de 20 años permite solucionar en gran parte problemas de adaptación de los pacientes a prótesis nuevas y debe permitir ejecutar estudios comparativos longitudinales con otras técnicas protésicas. A continuación se describe un caso clínico en el que se ilustran los procedimientos clínicos y de laboratorio involucrados en la duplicación de prótesis total.

\section{MATERIALES Y MÉTODOS}

Se utilizó un duplicador "Denture duplicator flask" fabricado en Colombia, similar al de la casa 3i, que consiste en una especie de mufla con dos secciones de aluminio que ajustan entre sí, con la ayuda de una bisagra intermedia y de un tornillo de cierre y ajuste en el otro extremo de la bisagra. El duplicador presenta orificios de escape y retención para el material de impresión en las bases $(11,12)$. 
Se utilizó silicona para copiar las prótesis totales superior e inferior del paciente, una a la vez, introduciendo siempre la superficie externa (no noble) en el material de impresión y dejando la superficie noble expuesta. En la segunda fase o de copiado, se cierra el duplicador y, una vez se obtiene la polimerización, se abre abrimos el duplicador y se realiza una copia de los dientes en parafina por escurrimiento. La base se copia en acrílico de autocurado para obtener un duplicado en cera y parafina cuyo fin es realizar el montaje en articulador con la copia acrílica y de cera.

Se estabilizan las bases (duplicados) en el paciente, se realizan los encofrados de cada prótesis duplicada, se toma la relación bicondilomaxilar y se monta el modelo superior $(13,14)$. Una vez adaptado el plato-base se toma la relación céntrica bien por método manual unidigital o bien en oclusión máxima cuando en el paciente coinciden la máxima intercupidación y la relación céntrica. Enseguida se reemplaza diente a diente sobre este encerado para ir completando progresivamente el enfilado y aumentar la dimensión vertical $3 \mathrm{~mm}$., que se determina previamente con el método de marcación extraoral en la parte más convexa de la nariz y el mentón. Después de realizar la prueba de oclusión y enfilado completo, se procede a tomar la impresión a boca cerrada con pasta zinquenólica. Se enmufla según la técnica convencional y se terminando el caso. El enfilado se ha ajustado a la técnica de oclusión bilateral balanceada. (15-18). Se obtiene así una copia casi exacta de toda la base protésica superior e inferior con dientes nuevos y oclusión adecuada.

\section{RESULTADOS}

FIGURA 1

PROCEDIMIENTO DE DUPLICACIÓN DE LAS PRÓTESIS 
A) CASO INICIAL. B) COPIA DE LA ZONA EXTERNA EN EL DUPLICADOR. C) COPIA DE LA ZONA NOBLE PROTÉSICA. D) PRÓTESIS DUPLICADA Y MONTADA EN EL ARTICULADOR. E) TOMA DE LA DIMENSIÓN VERTICAL OCLUSAL. F) INCREMENTO DE LA DIMENSIÓN VERTICAL VISTA LATERAL. G) ViSTA FRONTAL DEL ENFILADO TERMinAdO. H) PRUEBA DEL ENFILADO DUPLICADO EN BOCA. I) IMPRESIÓN DEFINITIVA A BOCA CERRADA. J) IMPRESIÓN INFERIOR Y PRÓTESIS ANTIGUA. K) CASO TERMINADO.

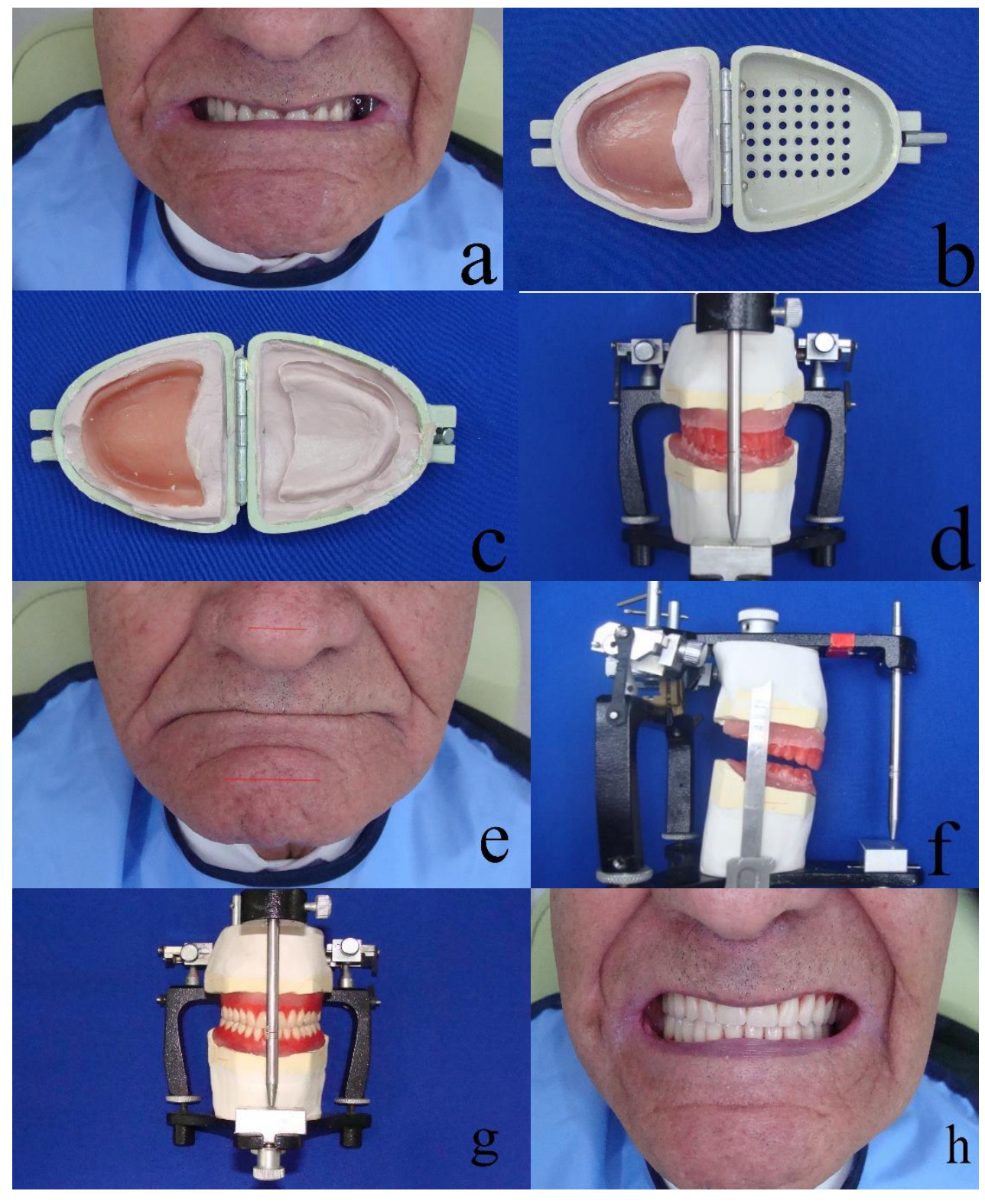




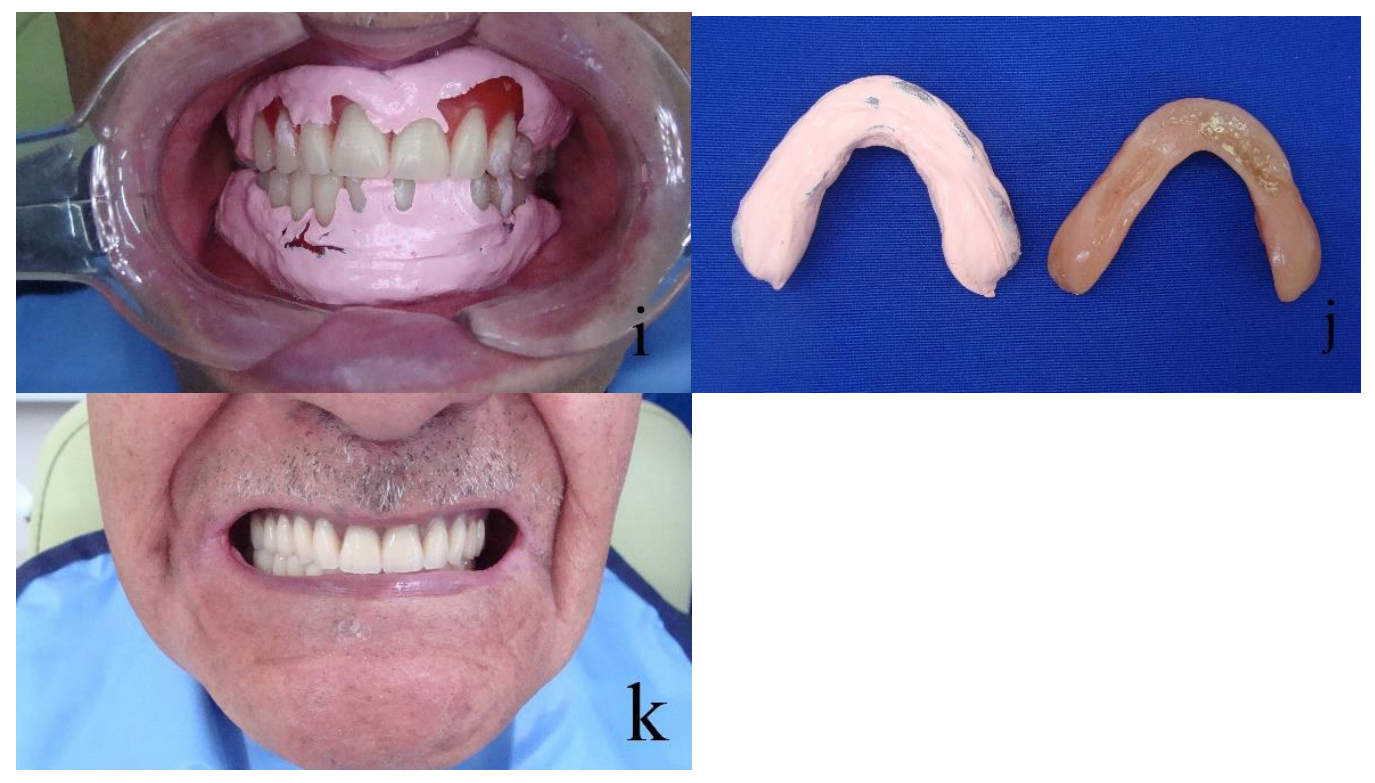

Esta es una técnica sencilla, cómoda, limpia para trabajar y efectiva en casos difíciles. Es práctica en pacientes que, por sus condiciones de desplazamiento, están recluidos en casa y por tanto la atención odontológica se brinda a domicilio. Esta técnica se puede considerar dentro de las técnicas simplificadas de elaboración de prótesis totales, ya que se pueden elaborar en tan solo tres citas clínicas. Cabe destacar que la prótesis duplicada sirve como plato base y los dientes como rodete. El sellado periférico y la impresión dinámica se logran con la impresión funcional directo en boca (19). El material aconsejado para obtener las prótesis duplicadas es la silicona debido a su menor distorsión y estabilidad dimensional (20).

La impresión final se realiza con boca cerrada, verificando la oclusión y la dimensión vertical. La toma de impresión final debe ser simultánea, ya que cuando se realiza separadamente en la misma sesión clínica aparecen mordidas abiertas. Es posible tomar las impresiones de cada arco por separado, pero cuando el antagonista ya está terminado y de esta forma las presiones y posiciones de las bases sobre 
los maxilares son simétricas y no cambian. El método de curado puede variar cuando se considere pertinente y en las citas de control no es necesario hacer ningún tipo de ajustes.

\section{DISCUSIÓN}

Se han desarrollado alternativas para elaborar prótesis totales, principalmente en los últimos años. Se busca mejorar el arsenal terapéutico para pacientes desdentados. Las técnicas clásicas han requerido alrededor de 10 citas clínicas, pero algunas técnicas alternativas han disminuido su número a 6 citas. Sin embargo, presentan dificultades de adaptación y estéticas y un buen número de controles después de la inserción. La técnica de duplicación de prótesis en termocurado es una alternativa que ofrece ventajas en menos números de citas, disminución del tiempo de laboratorio y disminución del tiempo clínico, siempre y cuando sea realizada con manos expertas. Se duplicaron las prótesis de un paciente con reabsorción avanzada de los rebordes y cuyas prótesis tenían 20 años de antigüedad. Se siguieron los pasos estandarizados de montaje, enfilado dentario y enmuflado. Se obtuvieron prótesis definitivas con éxito sin necesidad de ajustes durante las citas de control.

Esto corrobora la experiencia reportada por más de 20 años en el uso de esta técnica $(21,22)$. Se corroboró también la gran influencia que tienen los sensorreceptores y la importancia de mantener umbrales de estímulo similares a los que ha manejado el paciente por tantos años de uso protésico. Es importante considerar realizar duplicación protésica con las nuevas tecnologías de CAD-CAM, donde sería posible obtener copias exactas de las prótesis y por medio de escaneado, software y fresadora automatizada (23). 


\section{CONCLUSIONES}

Aunque se requieren ensayos controlados futuros y estudios longitudinales, se puede afirmar que algunas características que se pueden destacar de la técnica de duplicación de prótesis totales se encuentran: a) es una técnica simplificada; b) se puede emplear en pacientes discapacitados; c) es sencilla, rápida, eficiente y con resultados predecibles; d) se ahorra tiempo importante de laboratorio dental, dado que la prótesis duplicada actúa como plato base y cubeta de impresión simultáneamente; e) se ahorra tiempo de clínica al evitamos impresiones preliminares, recorte muscular, sellado periférico y selección de dientes; f) se aprovecha la adaptación muscular y los propioceptores que trae el paciente con sus prótesis antiguas; g) los fabricantes de duplicadores tradicionalmente elaboran copias en acrílico de autocurado, pero es posible obtener prótesis en materiales definitivos h) los costos de elaboración fueron menores; i) se puede realizar en tres sesiones clínicas; j) el número de controles es mínimo; k) facilita la selección de dientes y el enfilado, ya que se determina a partir de los dientes que está usando el paciente; 1) la técnica de impresión con boca cerrada mejora el ajuste final y se trabaja sobre la mucosa y resiliencia del paciente y no sobre un modelo de yeso.

\section{RECOMENDACIONES}

Desarrollar estudios longitudinales y ensayos clínicos aleatorizados para corroborar lo que se reporta aquí y en las referencias citadas.

\section{REFERENCIAS}


1. Matiz Cuervo J. Técnica para duplicación de prótesis total. Rev Asoc Colomb Gerontol Geriatr. 2001. 15(1).

2. Matiz Cuervo J. Duplicación de prótesis. México, México: El Manual Moderno; 2016.

3. Davis DM, Watson RM. A retrospective study comparing duplication and conventionally made complete dentures for a group of elderly people. Br Dent J. 1993 Jul 24; 175(2): 57-60.

4. Davenport JC, Heath JR. The copy denture Technique. Br Dent J. 1983; 155: 162-163.

5. Brill N, Tryde G, Schubeler S. The role of exteroceptors in denture retention. J Prosthet Dent. 1959; 9(5): 761-8.

6. Robinson JG. A denture copying technique when providing replacement dentures. J Dent. 1976; 4(1): 15-8.

7. Lindquist TJ, Ettinger RL. Patient management and decision making in complete denture fabrication using a duplicate denture procedure: a clinical report, J Prosthet Dent. 1999; 82: 499503.

8. Scott BJJ, Forgie AH, Davis DM. A study to compare the oral health impact profile and satisfaction before and after having replacement complete dentures constructed by either the copy or by the conventional technique. Gerodontol. 2006; (23): 79-86.

9. Lindquist TJ, Narhi TO, Ettinger RL. Denture duplication technique with alternative materials, J Prosthet Dent. 1997; 77: 97-8.

10. Heath JR, Basker RM. The dimensional variability of duplicate dentures produced by an alginate investment. Br Dent J. 1978; 144: 111-4.

11. Heath JR, Davenport JC. A modification of the copy denture technique. Br Dent J. 1987; 153: 3002. 
12. McCord JF, Hannah VE, Cameron D, Watson D, Donaldson AC. An update on the replica denture technique. Dent Update. 2010 May; 37(4): 230-2, 235.

13. Duthie N, Lyon FF, Sturrock KC, Yemm R. A copying Technique for replacement of complete dentures. Br Dent J. 1976; 144(8): 248-52.

14. Ansari IH. Duplicating an existing complete denture to make a replica. J Prosthet Dent. 1994; 72(4): 445-7.

15. Boos RH, Carpenter HO Jr. Technique for duplicating a denture. J Prosthet Dent. 1974; 31(3): 329-34.

16. Adam CE. Technique for duplicating an acrylic resin denture. J Prosthet Dent. 1958 ; 8(3) : 40610.

17. Koka S. Vertical dimension of occlusion. Int J Prosthod. 2007; 20(4): 342.

18. Misch C. Objective vs. subjective methods for determining vertical dimension of occlusion. Quintessence Int. 2000 Apr; 31(4): 280-2.

19. Zoeller GN, Beetar RF. Duplicating dentures. J Prosthet Dent. 1970; 23(3): 346-54.

20. Chamberlain JB, Basker RM. A method of duplicating dentures. Br Dent J. 1967; 122: 347-9.

21. Singer LI. The "zipper" technique for duplication dentures: Final impressions, replica dentures, and a complete denture splint. J Prosthet Dent. 1975; 33(5): 582-90.

22. Nassif J, Jumbelic R. Duplicated maxillary complete dentures. J Prosthet Dent. 1984; 52(5): 7559.

23. Kawahata N, Ono H, Nishi Y, Hamano T, Nagaoka E. Trial of duplication procedure for complete dentures by CAD/CAM. J Oral Rehab. 1997; 24: 540-8. 